\author{
Andre Nyberg ${ }^{1 \star}$, Britta Lindström ${ }^{1}$, Nils \\ Aronsson ${ }^{1}$, Magnus Näslund ${ }^{1}$ and Karin \\ Wadell $^{1,2}$ \\ ${ }^{1}$ Department of Community Medicine and \\ Rehabilitation, Physiotherapy. Umeå University, \\ Umeå, Sweden \\ ${ }^{2}$ Department of Public Health and Clinical Medicine, \\ Division of Medicine, Umeå University, Umeå, \\ Sweden \\ Dates: Received: 12 April, 2016; Accepted: 27 April, \\ 2016; Published: 28 April, 2016 \\ *Corresponding author: Andre Nyberg, Department \\ of Community Medicine and Rehabilitation, \\ Physiotherapy. Umeå University, 90187 Umeå, \\ Sweden, Tel: + 4690786 6639; Fax: + 4690786 \\ 9267; E-mail: andre.nyberg@umu.se \\ www.peertechz.com \\ ISSN: 2455-5487 \\ Keywords: Elastic bands; Knee extension; Maximal \\ strength; Older adults; One-repetition maximum; \\ Validity
}

\author{
Research Article
}

\section{Validity of using elastic bands to measure knee extension strength in older adults}

\section{Abbreviations}

1-RM: One-Repetition Maximum; ICC: Intraclass Correlation Coefficient; LoA; Limits of Agreement; R: Pearson's Correlation; R: Concordance Correlation Coefficients;

\section{Background}

Knee extension strength is an important component of physical fitness, it has an independent role in the prevention of chronic diseases and muscle weakness is strongly related to functional limitations and physical disabilities. Knee extension strength is also inversely associated with all-cause mortality, independent of confounding factors such as level of physical activity and cardiorespiratory fitness in both elderly as well as in people suffering from chronic diseases $[1,2]$. If the goal is to improve knee extension strength, resistance training programs are one of the most popular forms of exercise [3]. Resistance training is also recommended before other available exercise modalities as it is considered the most effective way to improve muscle strength and can be performed using a variety of exercise equipment [4].

One such exercise alternative is elastic resistance equipment such as elastic bands and tubing which has been found effective in increasing muscle strength in older adults $[3,5,6]$ offering an inexpensive and portable exercise equipment that is easy to use. It is also more accessible than other exercise equipment's such as isokinetic dynamometers or weight machines [7-9]. In a similar way as resistance training could be performed using different exercise equipment, evaluation of resistance training programs can be performed using a variety of measurement equipment, from uncomplicated functional tests to advanced laboratory tests [10-12]. Of importance is that the assessment of muscle function, in addition to being muscle groupspecific, also is heavily dependent on the type of contraction, velocity of movement and equipment used [13]. In a similar way as exercise training adaptations are specific to the stimulus applied, the most sensitive method of assessing exercise induced adaptations has been found when the method of assessment is as comparable as possible to the exercise modality used. Thus, the most sensitive method of assessing the effects of resistance training performed using elastic bands is an assessment method that also employs elastic bands [7]. In the assessment of muscle strength, the 1-repetition maximum (1-RM) test has been described as the "gold standard" for isotonic maximal muscle strength measurements [14] and is considered a reliable and valid measurement technique to evaluate knee extension strength in older adults using weight machines $[15,16]$. The validity of using elastic resistance bands to evaluate muscle strength in older adults has rarely been addressed, especially including a component of maximal effort such as a 1-RM test. It could be argued that the increase in the length of an elastic band, which occurs in any dynamic movement, 
will result in a progressive increase in resistance making accurate evaluations of 1-RM difficult $[17,18]$. However, the definition of 1-RM has previously been adopted with the use of elastic resistance bands [19] and our group have previously found that 1-RM using elastic bands is a valid method to measure shoulder flexion strength in older women and men [10].

To our knowledge, no previous study has investigated the use of 1-RM testing with elastic resistance bands to determine the maximal knee extension strength in older adults. The purpose of this study was to investigate the concurrent validity of a 1-RM test with elastic resistance bands in the measurement of knee extension maximal strength by evaluating the intraclass correlation coefficient (ICC) absolute agreement, correlations, and variations at an individual level using $95 \%$ limits of agreement (LoA) with isokinetic dynamometry testing as the reference standard. It was hypothesized that the elastic 1 -RM test would demonstrate good validity for the assessment of knee extension strength (ICC $\geq 0.61$ ).

\section{Material and Methods}

\section{Participants}

Twenty-four participants $(61.8 \pm 6.0$ years; $173.5 \pm 10.2 \mathrm{~cm}$; $71.9 \pm 15.7 \mathrm{~kg} ; 12$ women) were included in the study. Tests were performed in our exercise lab at the Department of Community Medicine, Physiotherapy, Umeå University, Umeå, Sweden.

To be eligible for participation in the study, participants had to be 50 years of age or older, without any current musculoskeletal disorders that would prevent them from performing a maximal concentric contraction during knee extension using their dominant (preferred) leg. The participants were asked not to perform strength training or any other activity that would physically exert their lower extremities within 24 hours leading up to the tests. The regional ethics board, Umeå, Sweden, approved the study (Reg. No. 2012-60-32M), and participants gave written informed consent prior to participation.

\section{Procedure}

Isokinetic dynamometry testing is thought to provide the most accurate assessment of muscular strength and has previously been used to determine the validity of using elastic resistance bands to measure muscle strength $[10,20,21]$. The participants performed concentric maximal contractions in an isokinetic dynamometer (KinCom ", Chattanoga Group, Inc) and 1-RM (that is, the maximum weight that could be lifted by a person in a single bout in the wholerange of movement) using elastic resistance bands (Thera-Bands, The Hygenic Corporation, Akron, OH, USA). At first, the participants were informed on the procedures of the tests. Thereafter, a five minute warm-up on an electronically braked cycle ergometer (Monark $818 \mathrm{E}$, ergomedic, Sweden ${ }^{\circ}$ ) at a submaximal load and cadence was performed. The Borg Scale of perceived exertion [22], was used and the participants were instructed to adjust the load between 11 and 13 on the scale with a cadence of 60 RPM, automatically controlled by the cycle ergometer.

The starting point of the two tests was set to $90^{\circ}$ of knee flexion, the end point for both tests was set to approximately five degrees below the maximum point of active knee extension for each participant. Standardized instructions and standardized verbal encouragement were given during both tests to facilitate a maximal effort [23], no visual feedback was provided. The same two test administrators (both male) conducted all tests, and both tests were performed during a single 60 minute visit for each participant (time of day: between 09:00-18:00). A pre-specified percentage of the peak torque value of $80 \%$ from the isokinetic test was used to determine the starting load for the 1-RM elastic band test. This meant that no randomization of exercise order was possible, resulting in each test session starting with the isokinetic test.

\section{Isokinetic assessment}

Isokinetic assessment of knee extension strength was performed with the dominant leg using a stationary dynamometer (Kin-Com , Chattanoga Group Inc) at an angular velocity of $60^{\circ} / \mathrm{s}$. Data were acquired using a PC and Kin-Com ${ }^{\star}$ software and digitally stored (12 bits, Mysas, Department of Biomedical Engineering \& Informatics, University Hospital, Umeå, Sweden). Isokinetic measurements of knee extension strength using a Kin-Com at $60 \%$ have previously demonstrated excellent test-retest, intra- and inter-rater reliability (ICC $=0.90,0.98$ and 0.93 , respectively) [11]. The test administrator performed a demonstration of the test before the participants were placed in the isokinetic dynamometer. The participants were fixated at the trunk, hip and distally on the tested leg with straps to minimize any compensatory body movements. The movement center of the isokinetic dynamometer was aligned with the lateral condyle (femur) of the tested leg. The participants were seated in the chair with the back rest at an angle of $100^{\circ}$. The seat was adjusted to a slight upward angle. The chair and dynamometer were positioned for knee extension movement assessment according to the specifications of the manufacturer with a standardized starting position (the starting point of the isokinetic dynamometer) of $90^{\circ}$ flexion of the knee joint (Figure $1 \mathrm{~A}$ ). The angular velocity was set to $60 \%$ s for both the concentric (knee extension) and eccentric phase (knee flexion) of the movement. The participants then performed two sets of five submaximal repetitions to familiarize themselves with the equipment and testing procedure and to acquire additional warm up [10,24]. At the time of the test the participants were instructed to exert themselves maximally during

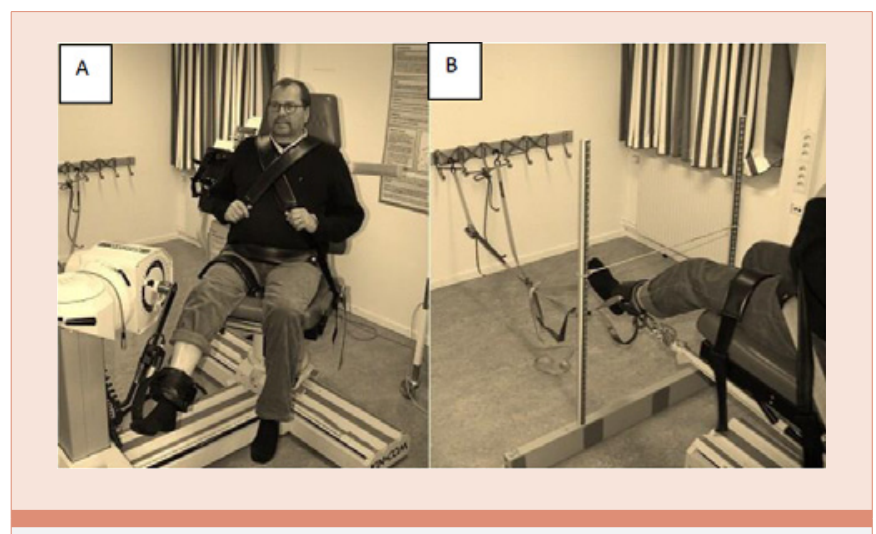

Figure 1: (A) Isokinetic test in a Kin-Com dynamometer; $(B)$ dynamic test using elastic resistance bands. 
the concentric phase and to relax during the eccentric phase. The test consisted of five maximal repetitions performed consecutively as previously used to measure 1-RM using elastic resistance bands in older adults [10]. The maximum value out of the five contractions were used to determine isokinetic peak torque.

\section{1-RM using elastic resistance bands}

The elastic resistance band test was performed using TheraBand elastic bands (Thera-band, The Hygienic Corporation, 1245 Home Ave. Akron, $\mathrm{OH} 44310$ ) in a seated position in the chair of the Kin-Com dynamometer. Custom made rings were fastened to both ends of the elastic bands. The elastic bands were fastened securely at ground level behind the chair using a carbine hook. This meant that the force trajectory was at a slight inclination from the point of origin on the floor to the point of insertion on the ankle. We used a modified ankle brace equipped with a carbine hook to fasten the elastic bands to the leg. The ankle brace was positioned distally on the tibia/fibula, just proximally of the medial/lateral malleolus, making sure the participants were able to perform a dorsal flexion of the foot with a full range of motion. Straps were fastened to the ankle brace and to the wall in front of the seat; this made sure that the straps supported the entire load from the elastic bands until the participants extended their knee from the starting position of 90 degrees of knee flexion (Figure $1 \mathrm{~B}$ ).

Five different elastic bands with increasing resistance were used in this trial; (yellow [thin] <, red [medium] <, green [heavy] <, blue [extra heavy] $<$, silver [super heavy]) [17]. Different elastic bands were combined to achieve an accurate loading which was based on the results from the isokinetic test (peak torque value) for each individual participant, respectively. The resting length of the elastic bands was set to $2 \mathrm{~m}$ and a ring was attached at both ends of the elastic band. A previous study has shown a linear progression of force after $50 \%$ elongation of elastic bands [17]. In this study, the bands were stretched to $100 \%$ of their resting length at the end of the range of motion. This was, with a measuring tape, ensured for each individual by measuring the distance from the point of origin to the point of insertion on the ankle brace when the knee was maximally extended. The length was adjusted by moving the seat of the isokinetic dynamometer backwards or forwards and the end point of maximal knee extension was controlled by a string tied horizontally between two vertical posts. During the test, participants were instructed to extend their leg until it touched the string (Figure $1 \mathrm{~B}$ ).

The resistance of each band was manually determined using a handheld dynamometer (Lutron fg 5100) through three steps. At first, each elastic band was pre-stretched to $100 \%$ of the original length 20 times before the tests started to stabilize the material [17]. Secondly, one end of the elastic band was fastened to a wall bar at floor level while the other end of the elastic band was fastened to the handheld dynamometer (also held at floor level). Carbine hooks were used to fasten the elastic band to the wall bar and the dynamometer, respectively. Finally, the elastic band was stretched by the test administrator to $100 \%$ elongation of its original length (i.e., from 2 to 4 meters) which was determined by a mark on the floor. The force registered by the handheld dynamometer at that mark was used to determine the resistance of each individual elastic resistance band. The second and the third (final) step was performed individually for each of the five (yellow, red, green, blue, silver) elastic bands used.

The following approach was used to determine 1-RM with elastic bands during concentric knee-extension:

1. Five knee extensions on $25 \%$ of isokinetic peak torque value was performed as warm-up and to familiarize with the elastic bands.

2. A minimum of one minute rest

3. One knee extension on $50 \%$ of isokinetic peak torque value.

4. A minimum of one minute rest

5. The start load for the elastic band 1-RM was set to $80 \%$ of isokinetic peak torque value (this was based on a priori pilot testing). If the participant succeeded to perform a knee extension throughout the whole range of motion on $80 \%$ of isokinetic peak torque the loading was increase by $5 \%$.

6. Step 5 was repeated until the participant reached his/her maximum load.

a. If the participant failed an attempt at a certain load he/she was given a second try at that load.

b. If the second attempt was failed their maximum load $(\mathrm{Kg})$ was determined to be the last load that was successfully performed [25].

c. The participants were given a minimum of one minute rest between each 1-RM attempt (step 5) to achieve adequate recovery [23].

\section{Statistics}

The following variables from the tests have been used in the analysis:

i) Maximum peak torque: the maximum value out of five contractions using isokinetic dynamometry testing at $60 \% \mathrm{~s}$ $(\mathrm{kg})$;

ii) Elastic resistance 1-RM: the highest load that could successfully be lifted throughout the whole range-of-motion in a single bout $(\mathrm{kg})$;

For statistical analysis the IBM Statistical Package for Social Sciences (SPSS) version 20.0 was used. Values are presented as mean and standard deviations (SD) or 95\% confidence intervals (95\%) if otherwise not stated. The normality of the distribution of the dependent variables of this study was tested with the Shapiro - Wilks test. Analysis of concurrent validity was conducted by comparing results from the elastic band 1-RM test to the gold standard laboratory-based isokinetic dynamometer test using ICC $_{2,1}$, Pearson's correlation (R) and concordance correlation coefficients $\left(\mathrm{R}_{\mathrm{c}}\right)$. The Pearson's correlation coefficient assesses association irrespective of magnitude of differences while both association and deviations from the line of identity $(\mathrm{y}=\mathrm{x})$ is assessed using concordance coefficient assessment. These measurements have previously been used to assess 
Table 1: Participant characteristics, isokinetic values, elastic resistance band one-repetition maximum (1-RM) and analysis of concurrent validity

\begin{tabular}{|c|c|c|c|c|c|c|c|c|}
\hline & \multicolumn{3}{|l|}{ Anthropometrics } & \multirow{2}{*}{$\begin{array}{l}\text { Elastic } \\
\text { 1-RM (kg) }\end{array}$} & \multirow{2}{*}{\begin{tabular}{|l} 
Isokinetic \\
Peak torque $(\mathrm{kg})$
\end{tabular}} & \multicolumn{3}{|c|}{ Validity } \\
\hline & Age (years) & Height (cm) & Weight (kg) & & & $\mathrm{R}$ & $\mathrm{R}_{\mathrm{c}}$ & $\mathrm{ICC}_{2,1}$ \\
\hline All & $61.8(59.3-69.4)$ & $173.5(169.2$ - 177.8) & $71.8(65.2-78.4)$ & $44.4(39.8-49.0)$ & $41.3(36.1-46.4)$ & $.90 \dagger$ & $.85(.70-.93) \dagger$ & $.86(.64-.95) \dagger$ \\
\hline Male & $61.8(57.2-66.5)$ & $180.8(176.9-184.8)^{\star}$ & $84.5(78.9-90.1)^{\star}$ & $52.0(46.0-58.0)^{*}$ & $48.8(40.9-56.7)^{\star}$ & $.85 \dagger$ & $.78(.46-.92) \dagger$ & $.80(.45-.94) \dagger$ \\
\hline Female & $61.8(58.9-64.8)$ & $166.2(161.2-171.2)$ & $59.1(53.3-64.9)$ & $36.8(33.0-40.7)$ & $33.7(30.3-37.0)$ & $.77 \dagger$ & $.62(.22-.85) \dagger$ & $.67(.11-.90) \dagger$ \\
\hline
\end{tabular}

Data is presented as mean and or $95 \%$ confidence intervals, if applicable. ${ }^{*} p<0.01$, women compared to men, $\dagger$ all $p<0.05$. ICC $=$ intraclass correlation coefficient, $\mathrm{R}=$ Pearsson correlation coefficient, $\mathrm{R}_{\mathrm{c}}=$ concordance correlation coefficient.

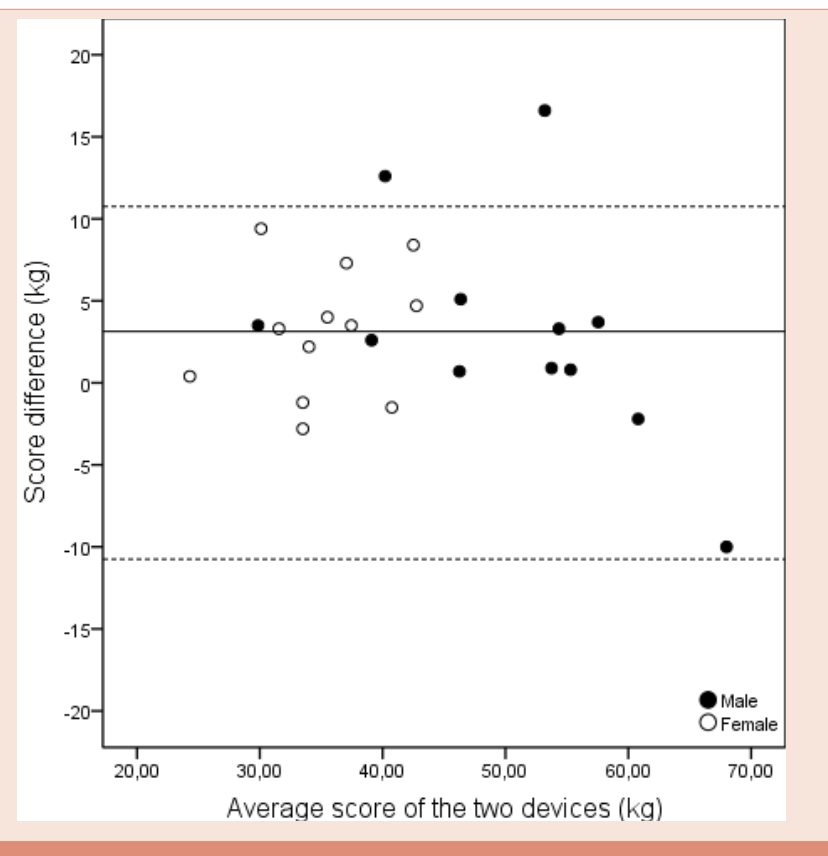

Figure 2: Bland-Altman plot of individual variation between isokinetic peak torque and elastic resistance band one repetition maximum (1-RM) with individual mean values between isokinetic peak torque and elastic resistance band 1-RM. Reference lines of the mean of the differences and \pm 1.96 SD (i.e., $95 \%$ limits of agreement). The mean of the difference was $3.1 \mathrm{~kg}$ and $95 \%$ limits of agreement was $10.5 \mathrm{~kg}$.

concurrent validity of knee extension strength using a Kin-Com [11]. In addition, the coefficient of determination $\left(\mathrm{r}^{2}\right)$ was utilized to indicate the percent of the total variance in peak torques explained by the 1-RM elastic resistance band scores. A standard BlandAltman plot with 95\% LoA was also calculated to examine individual variations [26]. This means that the mean values of isokinetic and the elastic resistance band 1-RM tests [isokinetic + elastic/2)] were plotted against the mean difference in individual force of the isokinetic and the elastic resistance band 1-RM [(difference = isokinetic - elastic)]. From the mean difference $(d)$, individual variation (i.e., $95 \% \mathrm{LOA}=$ $d+2 \mathrm{SD}_{\text {diff }}$ ) were calculated [26]. Significance level was set at $p<0.05$. Point estimates of the correlation and ICC values for validity analyses were interpreted as excellent $(\geq 0.81)$, good $(0.61-0.80)$, moderate $(0.41-0.60)$, or poor $(<0.40)$ [27].

\section{Results}

All participants completed both isokinetic and 1-RM tests. Four participants (two men and two women) stated their left leg as their dominant. Participant characteristics and results from the concurrent validity analysis are shown in Table 1, and individual variations (95\% LOA) are illustrated in Figure 2. Validity of 1-RM measurements of knee extension strengths were good to excellent and the 1-RM elastic band test explained $80 \%(\mathrm{r} 2=0.80)$ of the total variance in peak torques at $60 \%$ s. The average number of trials to reach elastic resistance band 1-RM was 7.5 (2.7) and the individual variation among women was $\pm 7.7 \mathrm{~kg}$ and among men $\pm 13.1 \mathrm{~kg}$.

\section{Discussion}

Elastic resistance band 1-RM testing demonstrated good to excellent validity to measure knee extension strength in older women and men, explaining $80 \%$ of the difference between measurement techniques. These results indicate that elastic resistance bands can be used to evaluate knee extension strength among older adults. This is of importance, considering that the assessment of muscular strength using elastic bands may be more sensitive to gains resulting from this type of training [7]. However, it is important to keep in mind that there are variations on an individual level, variations that to some extent could be explained by the greater freedom of movement allowed by the elastic bands in comparison to the isokinetic dynamometry [10].

To our knowledge this is the first study to use direct measurement of 1-RM of knee extension strength with elastic resistance bands and also the first study to evaluate the validity of knee extension strength assessment using elastic bands among older adults. Elastic resistance bands have previously been found valid and reliable in measuring knee extension strength in younger adults using multiple-RM testing or by predicting a $1-\mathrm{RM}$ from a multiple-RM test $[20,21,28]$. Taylor et al. [21], found an excellent correlation $(R=0.85)$ between a $8-R M$ test using elastic resistance bands and isokinetic peak torque values at $60 \%$ among young adults (mean age: $23 \mathrm{yrs}$ ), a test procedure that also was highly reliable (ICC $\geq 0.90)$ [28]. In a similar way, Guex et al. [20], found excellent validity $(\mathrm{R}=0.93)$ and relative reliability (ICC $=.99$ ) of elastic bands to evaluate knee extension strength in young adults (mean age: $31 \mathrm{yrs}$ ) by predicting knee extension 1-RM from a multiple-RM test of 10 repetitions or less. These results are in line with our observations indicating that elastic resistance bands are a valid tool to assess knee extension strength in both women and men. No adverse events occurred which is in line with previous studies using 1-RM measurements to measure knee extension strength in elderly, in which occurrence of adverse events are rare [29].

In comparison to previous studies [20,21] we also analyzed the concurrent validity separately for women and men. A lower agreement (lower ICC) was found for women compared to men 
and the mixed group results that are similar to what was previously observed during 1-RM testing of shoulder flexion strength among older adults [10]. As the variation among individuals will affect the ICC, i.e. a larger variation between individuals result in a higher ICC, the higher agreements among the men and in the mixed group could be explained by a greater range of force values (illustrated by the larger standard deviation) seen in these groups compared to women alone $[10,26]$.

The strengths of the study include the use of strict standardization of test procedures, execution of 1-RM testing in accordance with recommended guidelines [14] and the familiarization of both elastic band and isokinetic dynamometry tests in order to minimize potential learning effects [30]. Our study also had some limitations. It is recommended to reach $1-\mathrm{RM}$ in three to five attempts [14]. From a priori pilot testing, $80 \%$ of isokinetic maximum peak torque value was used as an initial load during the elastic band test. This, in addition to the decision to increase resistance with five percentages between each successful attempt, led to the majority of participants performing more than five attempts. This may have affected the results of the study due to exertion. Nevertheless, adequate rest was provided between attempts [23]. Further, in order to mirror test procedures, both tests were performed in a controlled setting with identical starting positions using the chair of the isokinetic dynamometry. In comparison to an ordinary chair that would be used in a clinical setting, the chair of the isokinetic dynamometry is higher resulting in a different trajectory of the elastic band to the exercising limb. As the joint loading on the muscle is affected by the angle of insertion of the elastic band we cannot conclude if similar relationship and differences would be obtained if using another sitting position [31]. Further, elastic 1-RM was consistently reported greater than isokinetic peak torque values. One possible explanation is that the string that marked the end point of knee extension in the elastic band test was visible for the participants (Figure $1 \mathrm{~B}$ ). This provided a visual feedback and gave the participants a visual goal to reach. Some of the participants expressed that they received a "boost" or increased motivation to reach the line, in comparison to the isokinetic test. Visual feedback has previously shown to increase grip-strength (6\% increase) compared to if no visual feedback was provided [32]. To counter this we could have used a screen to block out the visual feedback provided by the string from the participants. Another potential limitation is that we did not control the speed of motion during the 1-RM elastic band test. Participants often utilized a rapid contraction during the elastic 1-RM test in comparison to a slower contraction $\left(60^{\circ} / \mathrm{s}\right)$ during the isokinetic test. As differences in speed-of-motion seem to lead to differences in variation and reliability of knee extension measurements could it be hypothesized that the difference between measurement techniques could have been decreased if a similar speed of motion would have been used [33,34]. Finally, the study design prevented randomization of testing order that would have strengthened the design of the study since it may have reduced possible variations in motivation within the participant group [35].

\section{Conclusions}

The clinical implications of the findings of this study is that elastic resistance bands seem to be a valid tool for direct measurement of maximal knee extension strength in older women and men but with wide variations on an individual level. This offers an inexpensive and portable alternative in situations with no access to more expensive and sophisticated equipment, such as isokinetic dynamometers or weight machines that also may be more sensitive to change. Further research on the validity and reliability of using elastic resistance bands to assess different aspects of knee extension performance in older adults is warranted, preferable also involving older adults living with chronic diseases in which the use of elastic resistance bands to increase muscle performance is both effective and becoming more commonly used $[3,36]$.

\section{Acknowledgements}

We would like to thank the participants for their time and effort participating in this study. This study was supported by grants received from the Medical Faculty of Umeå University (223-335709). The funder had no role in study design, data collection, analyze, decision to publish, preparation or reporting of the study manuscript. Neither of the authors has any competing interest to declare.

\section{References}

1. Volaklis KA, Halle M, Meisinger C (2015) Muscular strength as a strong predictor of mortality: A narrative review. Eur J Intern Med 26: 303-310.

2. Swallow EB, Reyes D, Hopkinson NS, Man WD, Porcher R, et al. (2007) Quadriceps strength predicts mortality in patients with moderate to severe chronic obstructive pulmonary disease. Thorax 62: 115-120.

3. Martins WR, de Oliveira RJ, Carvalho RS, Damasceno VD, da Silva VZM, et al. (2013) Elastic resistance training to increase muscle strength in elderly: A systematic review with meta-analysis. Arch Gerontol Geriat 57: 8-15.

4. American College of Sports Medicine (2009) American College of Sports Medicine position stand. Progression models in resistance training for healthy adults. Med Sci Sports Exerc 41: 687-708.

5. Mikesky AE, Topp R, Wigglesworth JK, Harsha DM, Edwards JE (1994) Efficacy of a Home-Based Training-Program for Older Adults Using Elastic Tubing. Eur J Appl Physiol O 69: 316-320.

6. Topp R, Woolley S, Hornyak J, Khuder S, Kahaleh B (2002) The effect of dynamic versus isometric resistance training on pain and functioning among adults with osteoarthritis of the knee. Arch Phys Med Rehab 83: 1187-1195.

7. Manor B, Topp R, Page P (2006) Validity and reliability of measurements of elbow flexion strength obtained from older adults using elastic bands. J Geriatr Phys Ther 29: 18-21.

8. Hostler D, Schwirian Cl, Campos G, Toma K, Crill MT, Hagerman GR, et al (2001) Skeletal muscle adaptations in elastic resistance-trained young men and women. Eur J Appl Physiol 86: 112-118.

9. Colado JC, Triplett NT (2008) Effects of a short-term resistance program using elastic bands versus weight machines for sedentary middle-aged women. J Strength Cond Res 22: 1441-1448.

10. Nyberg A, Hedlund M, Kolberg A, Alm L, Lindström B, et al. (2014) The accuracy of using elastic resistance bands to evaluate muscular strength. European Journal of Physiotherapy 16: 104-112.

11. Mentiplay BF, Perraton LG, Bower KJ, Adair B, Pua YH, et al. (2015) Assessment of Lower Limb Muscle Strength and Power Using Hand-Held and Fixed Dynamometry: A Reliability and Validity Study. PLoS One 10: e0140822.

12. Ng SS, Cheung SY, Lai LS, Liu AS, leong SH, et al. (2015) Five Times SitTo-Stand test completion times among older women: Influence of seat height and arm position. J Rehabil Med 47: 262-266.

13. Saey D, Troosters T (2008) Measuring skeletal muscle strength and endurance, from bench to bedside. Clin Invest Med 31: E307-311. 
14. Thompson WR, Gordon NF, Pescatello LS (2010) ACSM's guidelines for exercise testing and prescription. (8thedn). Lippincott Williams \& Wilkins Philadhelpia, USA.

15. Schroeder ET, Wang Y, Castaneda-Sceppa C, Cloutier G, Vallejo AF, et al (2007) Reliability of maximal voluntary muscle strength and power testing in older men. J Gerontol A Biol Sci Med Sci 62: 543-549.

16. Verdijk LB, van Loon L, Meijer K, Savelberg HH (2009) One-repetition maximum strength test represents a valid means to assess leg strength in vivo in humans. J Sports Sci 27: 59-68.

17. Patterson RM, Stegink Jansen CW, Hogan HA, Nassif MD (2001) Material properties of Thera-Band Tubing. Phys Ther 81: 1437-1445.

18. Page P, Ellenbecker ST (2003) The scientific and clinical application of elastic resistance. Champaign, IL: Human Kinetics.

19. Melchiorri G, Rainoldi A (2011) Muscle fatigue induced by two differen resistances: Elastic tubing versus weight machines. J Electromyogr Kinesiol 21: 954-959.

20. Guex K, Daucourt C, Borloz S (2015) Validity and reliability of maximalstrength assessment of knee flexors and extensors using elastic bands. J Sport Rehabil 24: 151-155.

21. Taylor JD, Fletcher JP (2013) Correlation between the 8-repetition maximum test and isokinetic dynamometry in the measurement of muscle strength of the knee extensors: A concurrent validity study. Physiother Theory Pract 29: 335-341.

22. Borg GA (1982) Psychophysical bases of perceived exertion. Med Sci Sports Exerc 14: 377-381.

23. Parcell AC, Sawyer RD, Tricoli VA, Chinevere TD (2002) Minimum rest period for strength recovery during a common isokinetic testing protocol. Med Sci Sports Exerc 34: 1018-1022.

24. Lindström B, Waling K. Sundelin G, Ahlgren C (2003) Test-retest reliability of biomechanical output and subjective ratings of exertion in isometric and isokinetic shoulder forward flexion in healty adults. Advances in Physiotherapy 5: 169-178.

25. Desgorces FD, Berthelot G, Dietrich G, Testa MS (2010) Local muscular endurance and prediction of 1 repetition maximum for bench in 4 athletic populations. J Strength Cond Res 24: 394-400.

26. Bland JM, Altman DG (1990) A note on the use of the intraclass correlation coefficient in the evaluation of agreement between two methods of measurement. Comput Biol Med 20: 337-340.

27. Bartko JJ (1966) The intraclass correlation coefficient as a measure of reliability. Psychol Rep 19: 3-11.

28. Taylor JD, Fletcher JP (2012) Reliability of the 8-repetition maximum test in men and women. J Sci Med Sport 15: 69-73.

29. Shaw CE, McCully KK, Posner JD (1995) Injuries during the one repetition maximum assessment in the elderly. J Cardiopulm Rehabil15: 283-287.

30. Gaines JM, Talbot LA (1999) Isokinetic strength testing in research and practice. Biological research for nursing 1: 57-64.

31. Biscarini A (2012) Determination and optimization of joint torques and joint reaction forces in therapeutic exercises with elastic resistance. Medical engineering \& physics 34: 9-16.

32. Chkeir A, Jaber R, Hewson DJ, Hogrel JY, Duchêne J (2014) Effect of Different Visual Feedback Conditions on Maximal Grip-Strength Assessment. XIII Mediterranean Conference on Medical and Biological Engineering and Computing 2013. LM. Roa Romero, Ed., ed: Springer International Publishing 41: 1127-1131.

33. Ribeiro F, Lepine PA, Garceau-Bolduc C, Coats V, Allard E, et al. (2015) Testretest reliability of lower limb isokinetic endurance in COPD: A comparison of angular velocities. Int J Chron Obstruct Pulmon Dis 10: 1163-1172.

34. Hsu AL, Tang PF, Jan MH (2002) Test-retest reliability of isokinetic muscle strength of the lower extremities in patients with stroke. Arch Phys Med Rehabil 83: 1130-1137.

35. Frontera WR, Hughes VA, Dallal GE, Evans WJ (1993) Reliability of isokinetic muscle strength testing in 45- to 78-year-old men and women. Arch Phys Med Rehabil 74: 1181-1185.

36. Alison JA, McKeough ZJ (2014) Pulmonary rehabilitation for COPD: are programs with minimal exercise equipment effective? J Thorac Dis 6: 16061614.

Copyright: (c) 2016 Nyberg A, et al. This is an open-access article distributed under the terms of the Creative Commons Attribution License, which permits unrestricted use, distribution, and reproduction in any medium, provided the original author and source are credited. 